\title{
Stress Relief in Reconstruction
}

\author{
Claudia E. Bach, Margret Giesen, and Harald Ibach \\ Institut für Grenzflächenforschung und Vakuumphysik, Forschungszentrum Jülich, D-52425 Jülich, Germany \\ T. L. Einstein \\ Department of Physics, University of Maryland, College Park, Maryland 20742-4111
}

(Received 18 November 1996)

\begin{abstract}
We report on the first direct measurement of the change of the surface stress in the reconstruction of the $\mathrm{Au}(111)$ and the $\mathrm{Au}(100)$ surfaces. For both surfaces the reconstruction relaxes the intrinsic tensile stress, by $22 \%$ and $5 \%$, respectively. A discussion of the data on the Au(111) surface in the FrenkelKontorova model shows that the energy gain due to the surface stress is not quite large enough to make the reconstructed phase energetically favored without the formation of the secondary herringbone structure of the solitons. On the $\mathrm{Au}(100)$ surface, the gain in elastic strain energy is clearly insufficient to cause the surface to reconstruct. [S0031-9007(97)03238-9]

PACS numbers: 68.35.Bs, 47.20.Dr, 61.16.Ch, 82.45.+z
\end{abstract}

The (111) surfaces of the fcc transition metals Pt and $\mathrm{Au}$ and the (100) surfaces of $\mathrm{Ir}, \mathrm{Pt}$, and $\mathrm{Au}$ are known to reconstruct to surface layers of higher density [1]. On the (111) surfaces, the reconstruction involves a nonuniform compression of the surface along the [110] direction, with soliton-type domain walls between areas where surface atoms occupy fcc or hcp surface sites [2-6]. The reconstruction has been discussed frequently using the Frenkel-Kontorova (FK) model [7-10]: In the onedimensional (1D) version, a chain of atoms (representing the top layer) linked by springs with nearest-neighbor force constant $\varphi$ " and "natural" spacing $b$ is placed in a sinusoidal potential with amplitude $W / 2$ representing a rigid substrate with periodicity $a$. The prime feature of the reconstruction, the soliton domain wall, results from energy minimization. Further minimization of a (very small) strain energy associated with the anisotropy of the stress relief in the soliton reconstruction leads to a secondary ("herringbone") structure of the solitons [11]. Despite the general consensus that the soliton reconstruction is driven by the large tensile stress $[12,13]$ on the $\operatorname{Pt}(111)[4-6,14]$ and the $\mathrm{Au}(111)$ surfaces, there is no direct experimental evidence, e.g., by measurements on the stress relief in the reconstruction.

On the reconstructed (100) surfaces of $\mathrm{Ir}, \mathrm{Pt}$, and $\mathrm{Au}$, the surface atoms form quasi-hexagonal (hex) commensurate and incommensurate overlayers. The atom density in the surface layer is higher by $(20-25) \%$. From firstprinciples calculations, Fiorentini et al. [15] find the surface stress for the unreconstructed (100) surfaces of Ir, Pt, and $\mathrm{Au}$ to be even larger than for the (111) surfaces. Furthermore, the stress on the (100) surfaces of the $5 d$ fcc metals Ir, Pt, and Au is also significantly larger than for the (100) surfaces of the corresponding $4 d$ metals. They [15] identify the tensile stress as driving the reconstruction of the (100) surfaces on the $5 d$ metals. Indirect experimental evidence for stress relief in the reconstruction was obtained for the $\operatorname{Ir}(100)$ surface [16]. However, neither theory nor experiment could determine the change in the surface stress quantitatively. Without direct information about the actual amount of stress energy relieved in the reconstruction of the (100) surfaces, this interpretation of the origin of the reconstruction is questionable. We dispute it below.

In this Letter we report the first measurements of the change in surface stress which accompanies the reconstruction of both the $\mathrm{Au}(111)$ and the $\mathrm{Au}(100)$ surfaces. The stress measurements were performed in an electrochemical cell, with the crystals immersed in a $0.1 \mathrm{M} \mathrm{HClO}_{4}$ solution, using the cantilever bending method [17-19]; the bending was ascertained using a Besocke-type scanning tunnel microscope (STM), which also monitored the surface structure [20]. We exploit the fact that the reconstruction can be lifted by raising the electrochemical potential. Prior to the measurements, the $\mathrm{Au}$ single crystals were annealed at $800^{\circ} \mathrm{C}$ for $2 \mathrm{~h}$ in oxygen and for $1 \mathrm{~h}$ in argon, then cooled down in argon. To ensure that changes in the surface stress would occur only on one side of the sample, the bottom surface was covered with nail polish. Contamination during the measurements was minimized by placing the entire microscope with the electrochemical cell in an argon atmosphere.

The $\mathrm{HClO}_{4}$ solution was fed into the cell under potential control with the potential set to $-140 \mathrm{mV}$ vs standard calomel electrode (SCE). Under these conditions the surfaces are stabilized in their reconstructed phases. Figure 1 shows STM images of the surfaces. (Because of the less rigid sample mount, the noise is larger than typical.) On the (100) surface [Fig. 1(a)], the hexagonal incommensurate reconstruction is identified by parallel stripes. The reconstructed areas appear as islands of less than a monolayer height in the upper- and the lower-right parts of Fig. 1(a). A statistical analysis of virgin (100) surfaces 


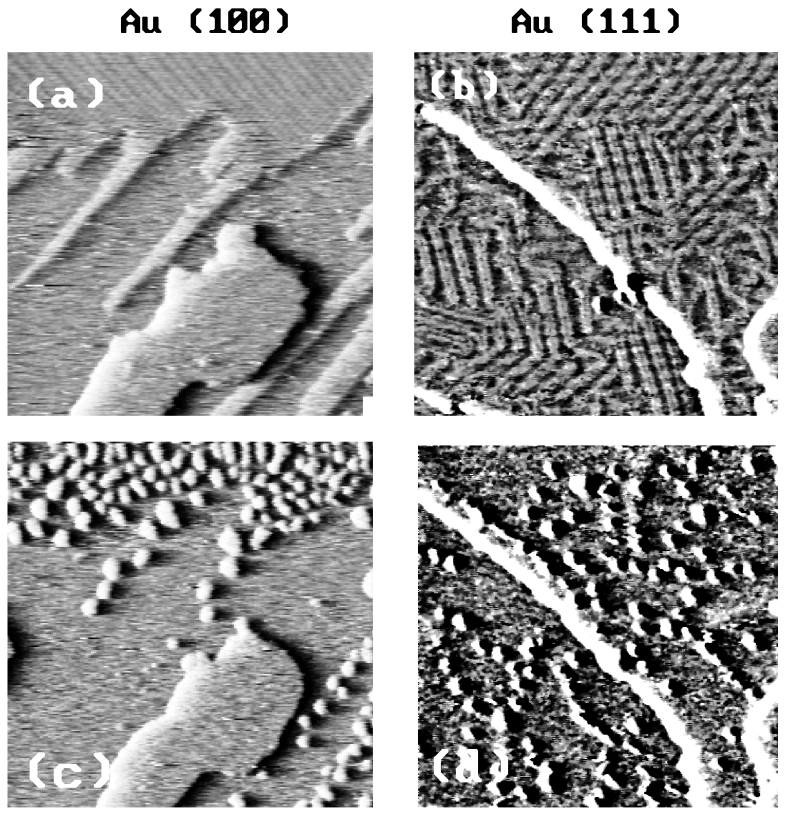

FIG. 1. STM images of $\mathrm{Au}(100)$ and $\mathrm{Au}(111)$ surfaces in $0.1 \mathrm{M} \mathrm{HClO}_{4}$. For the potential at $-140 \mathrm{mV}(\mathrm{a}, \mathrm{b})$, the surfaces are [partially] reconstructed. The reconstruction is lifted at a potential $>700 \mathrm{mV}(\mathrm{c}, \mathrm{d})$.

indicated that about $50 \%$ of the surface area was reconstructed, in approximate agreement with previous observations [21]. On the (111) surface [Fig. 1(b)], the soliton domain walls are clearly visible. On large enough terraces the solitons displayed the typical secondary herringbone structure [see upper part of Fig. 1(b)]. This structure is distorted near steps. On the (111) surface, about $70 \%$ of the surface was found to be reconstructed initially. For both surfaces the reconstruction is lifted when the potential is raised to positive values [21], and the extra atoms form islands on the surface at places that had been reconstructed [Figs. 1(c) and 1(d)]. Our STM images obtained as the potential became positive show that the lifting occurs first in areas where the reconstruction pattern is irregular in one way or another, e.g., near steps or near the edges of reconstructed areas [20]. In agreement with previous experiments [21], we found a partial restoration of the reconstruction after cycling the potential back to $-140 \mathrm{mV}$. After a few cycles, however, the surfaces do not reconstruct as the potential is cycled back. By comparing the stress versus potential curves in initial cycle with the later, stable cycles, we could determine the difference in the stress between the reconstructed and the unreconstructed surfaces at negative potentials. The results are presented in Fig. 2 for both the (100) and the (111) surface. The observed continuous decrease in the surface stress when the potential turns positive is due to an increasing number of specifically (i.e., chemically) adsorbed $\mathrm{ClO}_{4}^{-}$ions on the surface. The transformation into the $(1 \times 1)$ state is completed at $+900 \mathrm{mV}$ for either surface. There the surfaces must bear the same stress, regardless

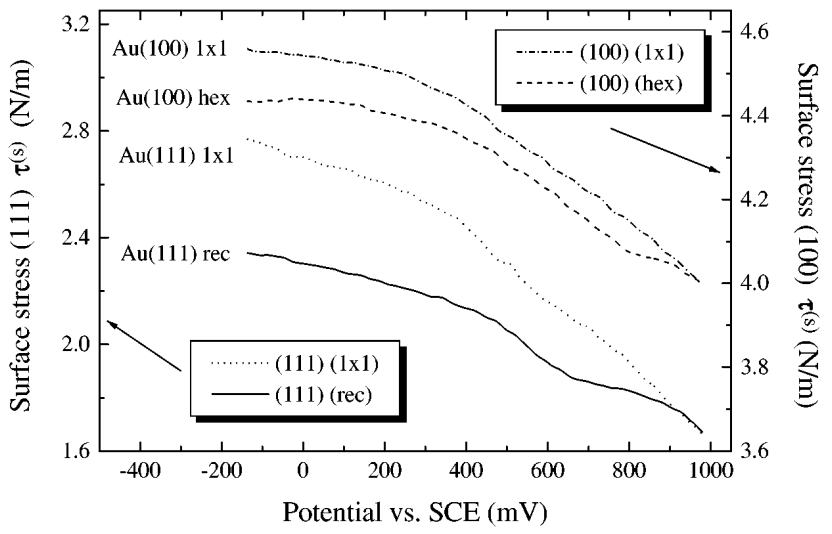

FIG. 2. Surface stress versus the potential for the initially reconstructed and unreconstructed surfaces of $\mathrm{Au}(100)$ and $\mathrm{Au}(111)$. All measurements are from negative to positive potentials.

of whether the surface was previously reconstructed at $-140 \mathrm{mV}$. The stress curves are therefore matched to this common reference point. For purposes of illustration, we have furthermore set the absolute value of the surface stress for the unreconstructed surfaces at $-140 \mathrm{mV}$ to match the $4.56 \mathrm{~N} / \mathrm{m}$ and $2.77 \mathrm{~N} / \mathrm{m}$ obtained by firstprinciples calculations for the $\mathrm{Au}(100)$ and $\mathrm{Au}(111)$ surfaces, respectively $[12,13,15]$.

Compared to the unreconstructed surfaces, the stresses for the reconstructed (100) and (111) surfaces are lower, at $-140 \mathrm{mV}$, by $0.12 \mathrm{~N} / \mathrm{m}$ and $0.43 \mathrm{~N} / \mathrm{m}$, respectively (Fig. 2). These numbers refer to surfaces which, as just noted, were only partially reconstructed initially. Assuming that the change in the macroscopically measured surface stress is proportional to the area which was reconstructed, we conclude-our main result - that the stress relaxation associated with reconstruction on the $A u(100)$ and the $\mathrm{Au}(111)$ surface is $-0.24 \mathrm{~N} / \mathrm{m}$ and $-0.61 \mathrm{~N} / \mathrm{m}$, corresponding to decreases of 5\% and $22 \%$, respectively. We estimate the error, due to the areal rescaling procedure and to other experimental uncertainties, to be $\pm 20 \%$ [22]. (Since the effect of the electrolytic fluid at small potential-where there is no specific adsorption-resembles that of physisorbed molecules, we expect little difference from surface stresses in vacuum.)

For both surfaces the reconstruction reduces the tensile stress. This is not surprising, since the average bond distances between the surface atoms are shorter in the reconstructed phases. The crucial issue is whether the reduction of the tensile stress and the corresponding gain in elastic energy is large enough to actually cause the reconstruction. Because of the different surface structures, this question must be addressed separately for the two surfaces.

As a first estimate for the elastic energy $\Delta u_{\mathrm{el}}$ released in the reconstruction of the $\mathrm{Au}(111)$ surface, we apply continuum theory to the stress-strain relation in the surface layer under the reasonable assumptions that only 
the surface layer is stressed and the macroscopic strain is 1D. We find [20]

$$
\Delta u_{\mathrm{el}}=2 \frac{1-\nu_{(111)}^{2}}{Y_{(111)} d_{(111)}} \tau^{(s)} \Delta \tau^{(s)},
$$

with $d$ the surface-layer thickness, $\nu$ and $Y$ the appropriate Poisson ratio and Young's modulus $[23,24]$. The surface stress $\tau^{(s)}$ is the bulk stress times $d$. The factor of 2 comes from the simplifying assumption that the measured stress is an average of uniaxial domains. After inserting elastic constants [23-25], $d_{(111)}$, the theoretically calculated $\tau^{(s)}$ for unreconstructed $\mathrm{Au}$, and the experimental $\Delta \tau^{(s)}$, we find $\Delta u_{\mathrm{el}}=-0.11 \mathrm{~N} / \mathrm{m}\left(=-6.9 \mathrm{meV} / \AA^{2}\right)$.

To corroborate our $\Delta \tau^{(s)}$, we can estimate it independently from the uniaxial compression in reconstruction:

$$
\Delta \tau^{(s)}=\frac{Y_{(111)} d_{(111)} \epsilon}{2\left(1-\nu_{(111)}^{2}\right)} .
$$

The strain $\epsilon$ is $-1 / N$, where $N$ is the length of the reconstructed unit cell in units of the nearest-neighbor distance $a$. Inserting elastic constants for $\mathrm{Au}$ [25] and using $N=22$ (so $\epsilon=-4.5 \%$ ), we calculate $\Delta \tau^{(s)}$ is $-0.59 \mathrm{~N} / \mathrm{m}$, close to the experimental value $-0.61 \mathrm{~N} / \mathrm{m}$.

To make the reconstructed phase energetically favorable, the gain in elastic energy must exceed the energy otherwise lost in the reconstruction. We estimate the net change using the 1D FK model. The energy for a $(1 \times 22)$ cell of the unreconstructed $(1 \times 1)$ phase is

$$
E_{(1 \times 1)} \equiv N \frac{\sqrt{3}}{2} a^{2} \gamma=N\left(\frac{3}{2} \varphi^{\prime \prime} a^{2} \delta^{2}+\Gamma\right),
$$

where $\gamma$ is the specific energy of the unreconstructed surface, $\delta \equiv(a-b) / a$ is the relative misfit, and $\Gamma$ is the energy to move an atom from the reservoir to the surface $[7,12,13]$.

The misfit can be expressed in terms of the surface stress on this unreconstructed surface as

$$
\tau^{(s)}=\sqrt{3} \varphi^{\prime \prime} \delta .
$$

Reconstruction alters the energy per $(1 \times N)$ cell by [7]

$$
\Delta E_{(\mathrm{cell})}=\Gamma+\frac{3}{2} \varphi^{\prime \prime} a^{2} \delta^{2}-\frac{3}{2} \varphi^{\prime \prime} a^{2} \delta+\frac{4 a}{\pi} \sqrt{\varphi^{\prime \prime} W},
$$

with $W$ the corrugation of the potential. (As noted at the outset, there are two minima per cell; the potential has periodicity $a / 2$ in the [110] direction, and the unreconstructed state is commensurate, of order $p=2$. However, the energy per length [Eq. (5)] is formally independent of $p$ [26].) From Eqs. (3)-(5) the change in the energy per area, $\Delta \gamma$, becomes

$$
\Delta \gamma=\left(\gamma-\tau^{(s)}+8 \sqrt{\varphi^{\prime \prime} W / 3 \pi^{2} a^{2}}\right) / N .
$$

The first two terms in this equation arise from the homogeneous contraction of the surface layer, while the third term is the contribution from the solitons. After insert- ing the calculated values for $\gamma$ and $\tau^{(s)}$ [12,13], one obtains for the energy of the homogeneous contraction $\Delta \gamma=$ $-4.32 \mathrm{meV} / \AA^{2}$, in reasonable agreement with $\Delta u_{\mathrm{e} 1}$.

The relief of the surface stress in a macroscopic measurement is the derivative of the energy change with respect to the area of the $(1 \times N)$ cell, i.e., using Eq. (6), $(2 a)^{-1} \partial\left(a^{2} \Delta \gamma\right) / \partial a$ :

$$
\Delta \tau^{(s)}=\left(\tau^{(s)}-\varphi^{\prime \prime} \sqrt{3}+8 \sqrt{\varphi^{\prime \prime} W / 3 \pi^{2} a^{2}}\right) / 2 N .
$$

To make estimates with these analytical expressions, one must choose appropriate values for the nearest neighbor force constant $\varphi^{\prime \prime}$ and the corrugation $W$. Particularly, the surface stress depends critically on the choice of $\varphi^{\prime \prime}$. Since the vibrational properties of gold are rather poorly described by a nearest-neighbor force-constant model [27], different matching procedures to experimental data yield rather diverse results. Furthermore, the longitudinal force constant between the $\mathrm{Au}$ surface atoms is significantly lower than the bulk value [28] and differs for the reconstructed and the unreconstructed surface [29]. The latter effect is beyond the scope of the FK model with its harmonic interaction potential. Because of these problems we choose the value of $\varphi^{\prime \prime}$ in the FK model as an effective coupling such that the experimentally observed change in the surface stress $\Delta \tau^{(s)}$ is reproduced and use this value to determine the energy gain in the reconstruction. The value of $\varphi^{\prime \prime}$ from the fit of $\Delta \tau^{(s)}$ depends only marginally on the choice for corrugation $W$. For $W=43 \mathrm{meV}$, which was used for a $2 \mathrm{D}$ simulation of the static and dynamic properties of the $\mathrm{Au}(111)$ surface [9], one obtains $\varphi^{\prime \prime}=18.1 \mathrm{~N} / \mathrm{m}$ (vs $\varphi^{\prime \prime}=17.1 \mathrm{~N} / \mathrm{m}$ for $W=0$ ). With these values for $\varphi^{\prime \prime}$ and $W$, and the first-principles values for $\gamma$ and $\tau^{(s)}$ $[12,13]$ inserted in Eq. (6), one obtains a small energy of $\Delta \gamma=0.013 \mathrm{~N} / \mathrm{m}\left(=0.8 \pm 0.5 \mathrm{meV} / \AA^{2}\right)$ [30], favoring the unreconstructed phase. The $62 \%$ error bars reflect the estimated $20 \%$ error bars in the experimental $\Delta \tau^{(s)}$.

About the same energy gain is associated with the formation of the secondary herringbone structure [11] as the loss $\Delta \gamma$. Thus, the secondary structure is probably necessary for the reconstruction to occur, at least for surfaces in UHV. For surfaces in an electrolyte, there is experimental evidence [21,31] that the herringbone secondary structure is not necessary. However, where the reconstruction has a secondary herringbone, it is more difficult to transform into a $(1 \times 1)$. In summary, it seems that the energy gain involved in the reconstruction of the $\mathrm{Au}(111)$ surface is very small, so that minute changes in the surface conditions can tip the balance to either side. Consistently, Huang [32] has observed that the reconstruction can also be lifted by very moderate bending of an $\mathrm{Au}(111)$ crystal in air.

For the $\mathrm{Au}(100)$ surface, the structural transformation is obviously beyond the scope of the FK model, so we cannot produce theoretical support for the experimental stress 
relaxation. Since stress does not quite produce the (111) reconstruction, it is implausible that the reconstruction of $\mathrm{Au}(100)$ is caused primarily by surface stress. The measured stress relaxation energy is barely a third that for the (111) surface. In contrast to the (111) case, the majority of surface atoms on a reconstructed (100) surface sit near bridge or even atop sites; the resulting energy cost is even higher because of the larger potential corrugation on (100) surfaces. The very nature of the reconstructed phase also suggests that maximizing the number of bonds is the most significant factor in the energy balance. This dominance of bond enhancement over stress relaxation was seen in the shifted-row reconstruction of $\mathrm{Pt}_{x} \mathrm{Ni}_{1-x}$ [33].

In summary we have found that the relaxation of the surface stress on the $A u(100)$ and the $A u(111)$ surface is $5 \%$ and $22 \%$ of the intrinsic stress, respectively. The elastic energy associated with the surface stress appears to be nearly large enough to cause the reconstruction of the $\mathrm{Au}(111)$ surface, but much too small to be the primary source of $\mathrm{Au}(100)$ reconstruction. More generally, it is now possible to measure the change in stresses associated with the reconstruction of surfaces. Electrochemical cells provide a fruitful way to manipulate the stress changes.

We gratefully acknowledge partial support of this work by the Fond der Chemischen Industrie of Germany (C.E.B., M. G., and H. I.), by the Alexander von Humboldt Foundation (M.G.), and by NSF-MRG DMR 9103031 and NSF-MRSEC DMR 96-32521 (T. L.E.).

[1] P. R. Watson, M. A. Van Hove, and K. Hermann, Atlas of Surface Structures (Am. Chem. Soc./AIP, New York, 1994), Vols. 1A and 1B; NIST Standard Ref. Database 42 (1995).

[2] J. Perdereau, J. P. Biberian, and G. E. Rhead, J. Phys. F 4, 798 (1974).

[3] V. Barth, H. Brune, G. Ertl, and R. J. Behm, Phys. Rev. B 42, 9307 (1990).

[4] K. G. Huang, D. Gibbs, D. M. Zehner, A. R. Sandy, and S. G. J. Mochrie, Phys. Rev. Lett. 65, 3313 (1990); A. R. Sandy, S. G. J. Mochrie, D. M. Zehner, K. G. Huang, and D. Gibbs, Phys. Rev. B 43, 4667 (1991).

[5] R. Sandy, S. G. J. Mochrie, D. M. Zehner, G. Grübel, K. C. Huang, and D. Gibbs, Phys. Rev. Lett. 68, 2192 (1992); G. Grübel, K. Huang, D. Gibbs, D. M. Zehner, A. R. Sandy, and S. G. J. Mochrie, Phys. Rev. B 48, 18119 (1993).

[6] M. Bott, Th. Michely, and G. Comsa, Phys. Rev. Lett. 70, 1489 (1993); M. Hohage, Th. Michely, and G. Comsa, Surf. Sci. 337, 249 (1995).

[7] M. Mansfield and R. J. Needs, J. Phys.: Condens. Matter 2, 2361 (1990).

[8] M. El-Batanouny, S. Burdick, K. M. Martini, and P. Stancioff, Phys. Rev. Lett. 58, 2762 (1987).

[9] R. Ravelo and M. El-Batanouny, Phys. Rev. B 40, 9574 (1989).
[10] N. Takeuchi, C. T. Chan, and K. M. Ho, Phys. Rev. B 43, 13899 (1991).

[11] S. Narasimhan and D. Vanderbilt, Phys. Rev. Lett. 69, 1564 (1992).

[12] R. J. Needs and M. Mansfield, J. Phys.: Condens. Matter 1, 7555 (1989).

[13] M. C. Payne, N. Roberts, R. J. Needs, M. Needels, and J. D. Joannopoulos, Surf. Sci. 211/212, 1 (1989).

[14] $\operatorname{Pt}(111)$ reconstructs only at higher temperature or with a high concentration of Pt adatoms.

[15] V. Fiorentini, M. Methfessel, and M. Scheffler, Phys. Rev. Lett. 71, 1051 (1993).

[16] S. Lehwald, J. G. Chen, G. Kisters, E. Preuss, and H. Ibach, Phys. Rev. B 43, 3920 (1991).

[17] R.E. Martinez, W.A. Augustyniak, and J.A. Golovchenko, Phys. Rev. Lett. 64, 1035 (1990).

[18] A. J. Schell-Sorokin and R. M. Tromp, Phys. Rev. Lett. 64, 1039 (1990).

[19] D. Sander and H. Ibach, Phys. Rev. B 43, 4263 (1991).

[20] H. Ibach, C. E. Bach, M. Giesen, and A. Grossmann, Surf. Sci. 375, 107 (1997).

[21] D. M. Kolb, Prog. Surf. Sci. 51, 109 (1996).

[22] The major source of error is systematic, coming from the conversion of measured strain to the deduced change in stress: since the sample is clamped along just one edge, the bending is neither pure $2 \mathrm{D}$ nor pure $1 \mathrm{D}$, though closer to the former. See Ref. [20] and H. Ibach, Surf. Sci. Rep. (to be published).

[23] W. A. Brantley, J. Appl. Phys. 44, 534 (1973).

[24] J. deLaunay, Solid State Phys. 2, 220 (1956).

[25] We use $s_{11}=2.33, s_{12}=-1.065$, and $s_{44}=2.38$, all in units of $10^{-12} \mathrm{~m}^{2} / \mathrm{N}$.

[26] P. Bak and V. J. Emery, Phys. Rev. Lett. 36, 978 (1976)P. Bak; , Rep. Prog. Phys. 45, 587 (1982).

[27] P.H. Dederichs, H. Schober, and D.F. Sellmaier, in Phonon States and Fermi Surfaces of Alloys, Landolt Börnstein 13a, edited by K.-H. Hellwege and F. L. Olsen (Springer, Berlin, 1981).

[28] R. B. Doak, U. Harten, and J. P. Toennies, Phys. Rev. Lett. 51, 578 (1983); V. Bortolani, A. Franchini, F. Nizzoli, and G. Santoro, Phys. Rev. Lett. 52, 429 (1984).

[29] X.-Q. Wang, Phys. Rev. Lett. 67, 1294 (1991).

[30] In a previous FK-model calculation [7] of the energy change due to reconstruction of $\mathrm{Au}(111)$, their nearestneighbor force constant (" $\mu^{\prime \prime ") ~ i s ~} 75 \mathrm{~N} / \mathrm{m}$, about quadruple ours. Most likely this factor enters during the scaling from $p=1$ to $p=2$, i.e., $a^{\prime}=2 a$ in their language.

[31] A. S. Dakkouri, Ph.D. thesis, University of Ulm (1996).

[32] Lin Huang, Ph.D. thesis, University of Bonn (1996); L. Huang, P. Zeppenfeld, and G. Comsa (to be published).

[33] M. Schmid, A. Biedermann, S. D. Bomig, P. Weigand, and P. Varga, Surf. Sci. 318, 289 (1994). While the authors state that the reconstruction does not change the number of bonds, since the increase in surface coordination is compensated by decreased coordination with the second layer, we note that the bonds in the top layer are new while the decrease in interlayer bonds comes from shifting from four-fold to bridge sites, a much smaller energy. 